\title{
Publisher Correction: A synthesis of behavioural and mainstream economics
}

Robert J. Aumann (D)

Correction to: Nature Human Behaviour https://doi.org/10.1038/s41562-019-0617-3, published online 27 May 2019.

In the version of this article initially published, the sentence beginning, "And while Smith does not say ..." should have referred to footnote 8 rather than function 8 . The error has been corrected in the HTML and PDF versions of the article. 